\title{
RCC - RESÍDUOS DA CONSTRUÇÃO CIVIL - CARACTERIZAÇÃO DE SUA GESTÃO INTEGRADA: UM ESTUDO DE CASO A ÁREA URBANA DE GARANHUNS-PE
}

\author{
Rodrigo Cordeiro da Silva ${ }^{1}$
}

Krystal de Alcântara Notaro²

\begin{abstract}
RESUMO
O Conselho Nacional do Meio Ambiente - CONAMA em suas atribuições estabelecem diretrizes, critérios e procedimentos para a gestão de resíduos da construção civil. Tal administração possibilita uma política ambiental mais ampla e eficaz, com a finalidade de alcançar, estabelecer e organizar a não geração, a redução, a reutilização, a reciclagem e o destino final dos materiais deixados do processo de construção. Tendo em vista que esses setores devem traçar metas, que cumpridas minimizam os impactos ao meio ambiente, aumentando assim sua produtividade e consequentemente seus lucros, priorizando uma política ecologicamente correta. O referido trabalho tem como objetivo fazer uma análise que mostra a complexa situação que se encontra o município de Garanhuns-PE, no tocante sobre a Gestão desses Resíduos. A investigação foi feita na área urbana de Garanhuns através de metodologia quantitativa, de acordo com números fornecidos pela Secretaria de Obras. Os dados e cálculos revela o custo que é pago pela Prefeitura Municipal para coleta, transporte e destino final (nem sempre adequado). Este estudo antecede questionamentos e conclui com a elaboração das seguintes questões: 1. Até que ponto as empresas de coleta e transporte contribuem para a Gestão integrada e sustentável dos RCC? 2. Quais os desafios que comprometem a Gestão sustentável dos RCC? 3. De que maneira o atual modelo de operação e Gestão das empresas de coleta e transporte lida com esse desafio? Estas interrogações traduz o cenário municipal e faculta resultados a proposto dos objetivos desta pesquisa quando se trada da Política de Gestão de RCC.
\end{abstract}

PALAVRA-CHAVE: Meio Ambiente, Gestão de RCC, Políticas Públicas

\footnotetext{
${ }^{1}$ Graduando de Bacharelado em Engenharia Civil, Autarquia do Ensino Superior de GaranhunsAESGA/Faculdade de Ciências Exatas de Garanhuns - FACEG. cordeirorodrigo13@hotmail.com. ${ }^{2}$ Profa M.s Krystal de Alcântara Notaro, Autarquia do Ensino Superior de Garanhuns AESGA/Faculdade de Ciências Exatas de Garanhuns - FACEG. krystal.notaro@gmail.com.
} 


\title{
RCC - WASTE OF CONSTRUCTION - INTEGRATED MANAGEMENT OF ITS CHARACTERISTICS: A CASE STUDY AREA URBANA GARANHUNS-PE
}

\begin{abstract}
The National Council of the environment - CONAMA on its attributions lay down guidelines, criteria and procedures for the management of waste from the civil construction. Such administration enables a wider and effective environmental policy, with the aim of achieving, establish and organize the next generation not, reduction, reuse, recycling and the final destination of the materials left the building process. In order that these sectors should establish targets, which met minimizing the impacts on the environment, thus increasing its productivity and consequently its profits, prioritizing an ecologically correct policy. The work aforementioned has as objective to make an analysis that shows the complex situation that is the municipality of Garanhuns-PE, in respect on the management of such waste. The research was conducted in the urban area of Garanhuns through quantitative methodology, according to figures supplied by the Secretariat of Works. The data and calculations reveals the cost that is paid by the Municipal Administration for collection, transportation and final destination (not always adequate). This study precedes questionings and concludes with the preparation of the following issues: 1. until extent the collection and transportation companies contribute to integrated and sustainable management of the RCC? 2. What are the challenges that compromise the sustainable management of RCC? 3. how does the current model of operation and management of companies of collection and transportation deals with this challenge? These questions translates the municipal scenario and provides the proposed results of the objectives of this research when entry of the Management Policy of RCC.
\end{abstract}

\section{RCC - RESIDUOS DE CONSTRUCCIÓN - INTEGRADO DE GESTIÓN DE SUS CARACTERÍSTICAS: UN CASO DE ESTUDIO ZONA URBANA GARANHUNS-PE}

\begin{abstract}
RESUMEN
El Consejo Nacional del Ambiente - CONAMA en sus cometidos de establecer directrices, criterios y procedimientos para la gestión de residuos de la construcción. Dicha administración permite a una política medioambiental más amplia y eficaz, con el fin de alcanzar, establecer y organizar la no generación, reducción, reutilización, reciclaje y disposición final de materiales dejado el proceso de construcción. Dado que estos sectores deben establecer metas, que se reunió minimizar los impactos sobre el medio ambiente, lo que aumenta su productividad y en consecuencia sus ganancias, dando prioridad a una política de medio ambiente. Este trabajo tiene como objetivo hacer un análisis que muestra la situación compleja que es la ciudad de Garanhuns-PE, en cuanto a la gestión de estos residuos. La investigación se realizó en el área urbana de Garanhuns a través de la metodología cuantitativa, según las cifras proporcionadas por el Departamento de Obras. Los datos y los cálculos revelaron el costo que se paga por la ciudad para la recolección, transporte y disposición (no siempre adecuada). Este estudio es anterior a las preguntas y concluye con la preparación de las siguientes preguntas: 1. ¿En qué medida las empresas de recogida y transporte contribuyen a la gestión integrada y sostenible de los RCC? 2. ¿Cuáles son los desafíos que comprometen la gestión sostenible de los RCC? 3. ¿Cómo funciona el modelo de funcionamiento actual y la gestión de la recogida y transporte se refiere a este desafío? Estas preguntas reflejan el entorno municipal y
\end{abstract}


proporciona resultados de los objetivos propuestos en esta investigación cuando Trada la política de gestión RCC.

PALABRA CLAVE: Medio Ambiente, Gestión de RCC, Políticas Públicas

\section{INTRODUÇÃO}

Com a Resolução CONAMA № 448, de janeiro de 2012, alterando o dispositivo da Resolução oㅜ 307/2002, em seu art. 1ำ estabelece de fato as diretrizes, os critérios e procedimentos para a gestão dos resíduos de construção civil, considerando alguns conceitos: a ABNT em sua norma NBR - 10004(2004) relata "resíduos Sólidos - Classificação", os resíduos da construção civil (RCC), também conhecidos como resíduos da construção e demolição (RCD) são em geral classificados como inertes (Classe II-B), uma vez que, quando submetidos a testes de solubilização não apresentam nenhum de seus componentes solúveis.

O gerenciamento e a administração desses resíduos é o que possibilita uma política ambiental e social mais ampla e eficaz. Por entender a Engenharia Civil como uma área de grande atividade, nos mais diversos ramos (construção, tecnologia, indústria, mercado e produção), gerando desenvolvimento econômico, social e político, mais, transformando o meio ambiente. Devida a isto a Construção Civil acaba se tornando a grande "vilã" do meio ambiente, seja pelo consumo excessivo dos recursos ambientais, ou pela modificação do ambiente, ou ainda pela geração de resíduo, o que causa um quantitativo excessivo de entulhos nas cidades.

É com esse objetivo que as novas políticas de gerenciamento de resíduos e de gestão integrada buscam obter, estabelecer e organizar a geração, a redução, a reutilização, a reciclagem e o destino final adequado desses resíduos. Dentro deste âmbito as políticas do meio ambiente em seu discurso trata dos 3R's da sustentabilidade, REDUZIR - adquirir bens e serviços de acordo com a necessidade da obra, evitando assim o desperdício; REUTILIZAR - fazer uso de matérias não utilizado para outros fins; RECICLAR - transformar restos de materiais em matériaprima para voltar ao ciclo produtivo, (CUNHA Jr, 2005). Essa política visa 


\section{Revista Nacional de}

Gerenciamento de Cidades

estabelecer uma relação mais harmônica entre consumidor e Meio Ambiente, diminuindo assim, o custo de vida e favorecendo o desenvolvimento econômico.

A importância de se ter conhecimento e gerir uma politica pública voltada para a coleta de resíduos devem ser uma realidade nos municípios, a implantação dessa política deve estimular o aproveitamento desse material, bem como favorecer a implantação de uma rede de áreas públicas ou privadas adequadas para a deposição regular dos mesmos. A criação dessa rede facilitaria uma possível triagem permitindo uma redução significativa da massa de resíduos a ser disposta no aterro ou nas encostas da cidade.

\section{OBJETIVOS}

Todo esse processo de coleta, transporte e destino adequado de resíduos possibilita geração de renda e diminuição de custos por parte do município. Associações de catadores se beneficiariam com a triagem desse material e 0 município passaria a utilizar parte dos resíduos como agregado reciclado na construção de pequenas obras e manutenção de vias.

O objeto desta pesquisa é despertar a sociedade e o poder público para os problemas que a não gestão desse material e apossível reciclagem pode mudar o cenário de Garanhuns-PE, tendo como referenciais conceitosda Resolução CONAMA e a analise dos dados colhidos no Município.

O que se propõem investigar, preliminarmente, com essas indagações é a correlação entre a Gestão integrada e sustentável dos RCC, em seus aspectos sociais, ambientais e econômicos, perguntas podem ser pensadas visando despertar os gestores municipais para tais indagações, até que ponto as empresas de coleta e transporte contribuem para a Gestão Integrada e Sustentável dos RCC, quais os desafios que comprometem a Gestão Sustentável desse material e de que maneira o atual modelo de operação e Gestão das empresas de coleta e transporte lida com esse desafio. 
Revista Nacional de Gerenciamento de Cidades

\section{METODOLOGIA}

A pesquisa foi desenvolvida na área urbana da cidade de Garanhuns-PE, imagem 1, no período de 2013 e 2014, através de metodologia quantitativa e descritiva, em razão do objeto da investigação. Segundo Cervo (2007), este tipo de pesquisa ocorre quando se registra, analisa e correlaciona fatos ou fenômenos, sem manipulá-los.

Imagem 1: Localização da área urbana de Garanhuns-PE.

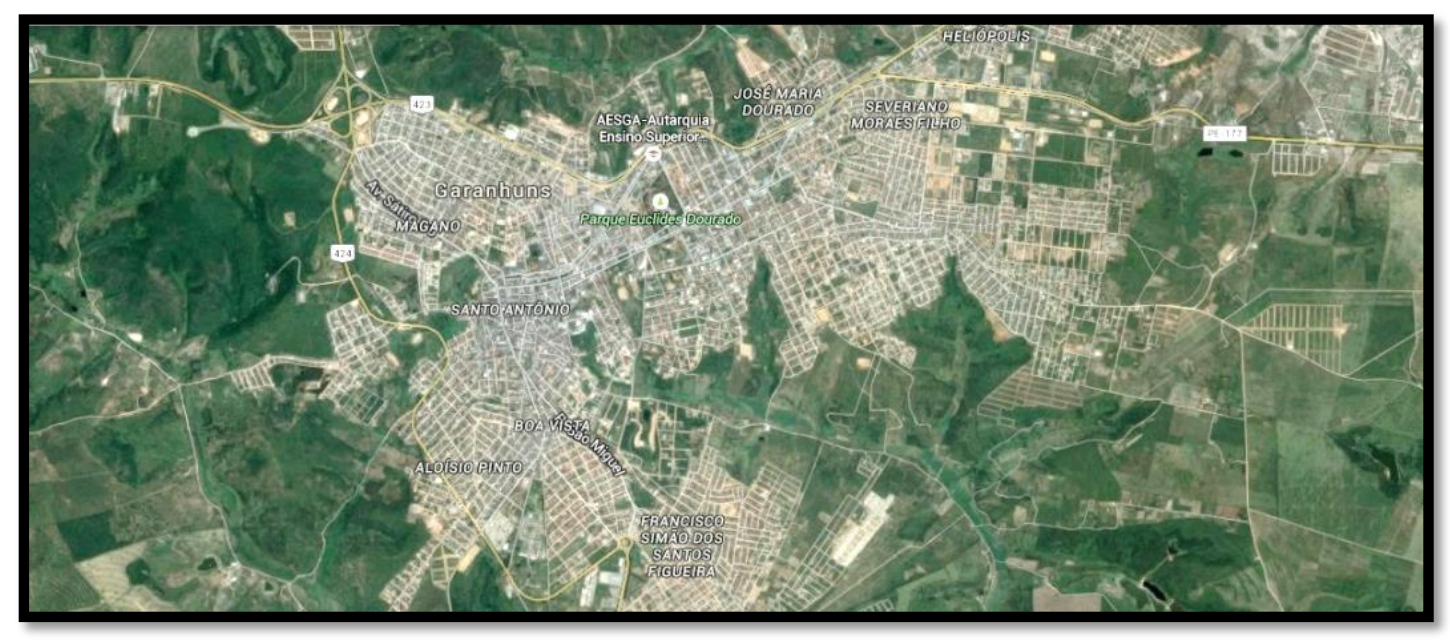

Fonte: Google maps, 2015.

Em pesquisa realizada pelo IBGE (Instituto Brasileiro de geografia e estatística) em 2013 a população estimada para o município de Garanhuns era de 135.138 habitantes e em 2014 essa população será de 136.057 habitantes, localizados em uma área territorial de $458,552 \mathrm{Km}^{2}$ e densidade demográfica de 282,21 hab/Km², IBGE 2010.

Para fundamentação da pesquisa foram utilizados estudos integrados e complementares. O referencial teórico teve como base na Resolução CONAMA, conferidas pela Lei no 6.938, de 31 de agosto de 1981, regulamentada pelo Decreto no 99.274, de 06 de junho de 1990; pesquisa bibliográfica através da análise de 


\section{Revista Nacional de}

Gerenciamento de Cidades

pesquisa documental de artigos científicos; sites; livros específicos e artigos de revistas e observação em campo.

Em seguida se realizou o estudo de caso, de acordo com Cervo (2007), trata-se de pesquisa sobre determinado indivíduo, família, grupo ou comunidade, para analisar aspectos variados sobre sua vida. O estudo de caso objetiva desenvolver declarações teóricas sobre a harmonia do processo e estruturas sociais

A coleta de dados foi possível através de memorial de cálculo, fornecidos pela Secretaria de Obras de Garanhuns, os quantitativos foram analisados e computados, de acordo com o número de caçambas, a quantidade de material transportado em toneladas $(t)$ e $o$ valor por cada $t$ colhida durante 24 meses de amostragem. A compilação dos dados foi realizada no Excel e a avaliação dos mesmos resultou em uma análise quantitativa do material durante o processo de investigação.

A empresa responsável pela coleta, transporte e destino dos resíduos é uma terceirizada pela Prefeitura de Garanhuns, a mesma, dispõe de três caçambas que fazem o trabalho diariamente. Os dados ao final de cada quinzena são computados, totalizando o quantitativo de toneladas que cada caçamba coleta no período.

O processo metodológico para base de resultado se deu da seguinte forma:

1. Quantificar e comparar o montante de RCC gerados em Garanhuns com a totalidade da região - para tal evento foi analisado graficamente em Excel o montante de caçambas, toneladas e custo do município com dados regionais;

2. Observar sua viabilidade em custos por $t$, onde se o mesmo for tratado com uma política de gerenciamento ambiental adequada - a atividade está diretamente ligada ao procedimento anterior, os dados fornecidos pelos gráficos darão subsídios para elaboração de um quantitativo em reais dessa atividade. 


\section{RESULTADOS}

\section{Quantificação de RCC}

Para quantificação da geração de RCC no município de Garanhuns-PE, foi colhidas informações via secretaria de obras do quantitativo em $t$ coletadas $\mathrm{e}$ transportadas pela empresa terceirizada nos anos de 2013 e 2014 . O volume médio desse material coletado diariamente é de 43.225t, totalizando um montante de 1.296.750t/mês em média para os anos avaliados, como podemos observar na figura 1 a seguir.

Figura 1: Produção de RCC no período.

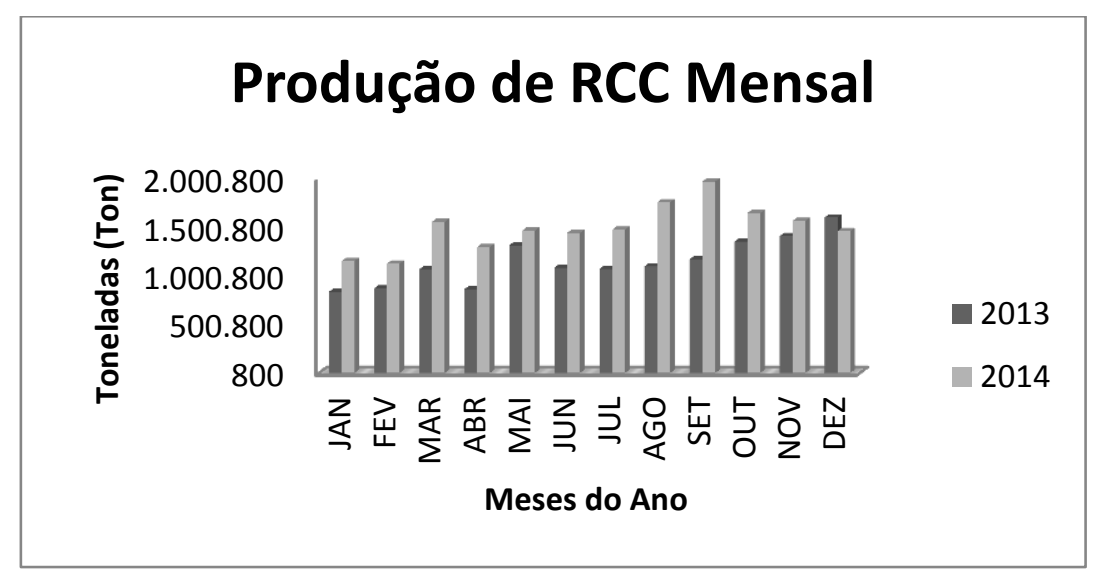

Fonte: Secretaria de Obras de Garanhuns-PE, (2014). Adaptação SILVA, R.C. (2015).

No gráfico se observa que a produção de resíduos apresenta-se maior nos meses de agosto a outubro 2014. Nesses meses verifica-se uma produção média de resíduos da ordem de 1.786,88t/mês, isso se deve ao fim do inverno onde o índice de demolição é maior e início do verão, nesse período os indicadores acima comprovam essa estatística.

O gráfico da figura 2 faz o mesmo comparativo, desta vez anual da geração de RCC em Garanhuns-PE. Foi observado um aumento significativo no ano de 2014 
em relação a 2013, esse aumento percentual na produção foi na ordem de $23,3 \%$, isso se deu ao crescimento na economia nacional nesse período e ao elevando mercado da construção civil.

Figura 2: Produção de RCC anual

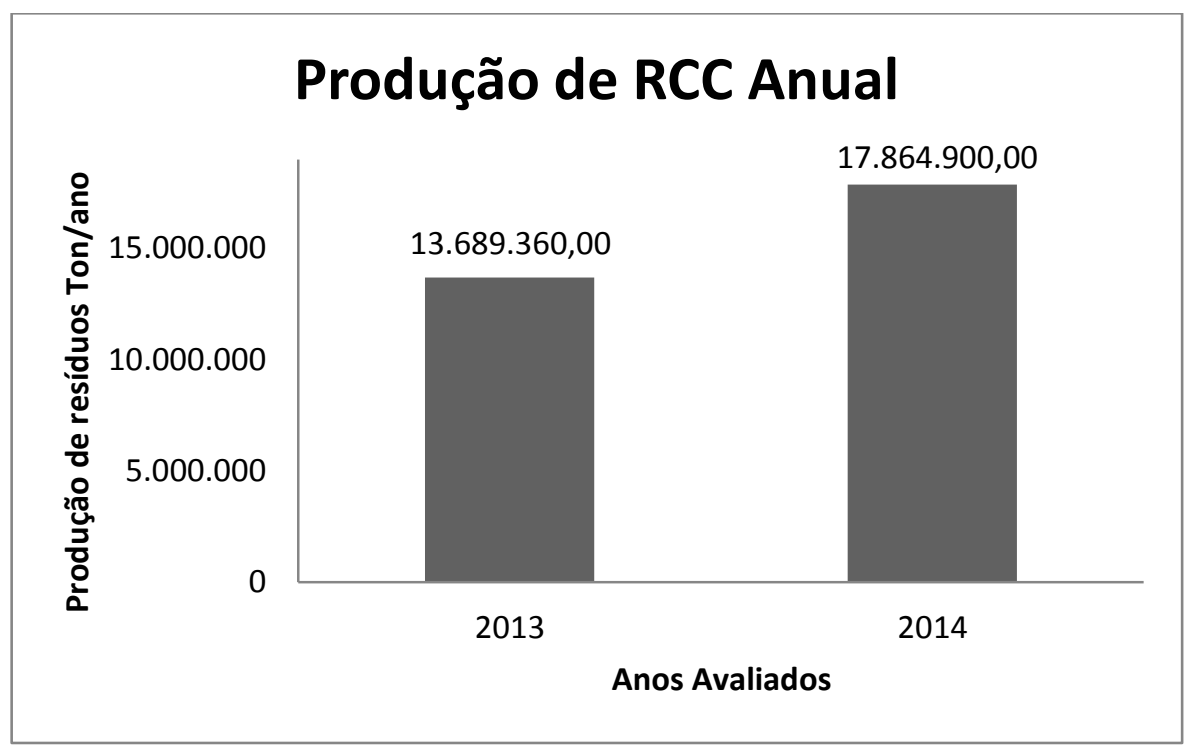

Fonte: Secretaria de Obras de Garanhuns-PE, (2014). Adaptação SILVA, R.C.(2015).

Realizado um comparativo da geração de RCC produzidos em GaranhunsPE com dados da Região Nordeste os índicesmostra que no ano de 2012 a população da região era 55.794.707 hab. e tinha uma coleta diária de 22.162.000t de RCC com uma produção per capta de 0,397Kg/hab/dia, (Associação Brasileira de Empresas de Limpeza Pública e Resíduos Especiais - ABRELPE, 2013).

Os dados para Garanhuns demonstram que o município apresenta uma população de 135.138 habitantes e coleta diária de 37.505 toneladas resultando em uma produção per capta de $0,278 \mathrm{Kg} / \mathrm{hab} / \mathrm{dia}$, ou seja, $1,07 \%$ da coleta diária do Estado. Como mostra a tabela 1. 
Tabela 1: Indicadores da coleta de RCC.

\begin{tabular}{cccc}
\hline & POPULAÇÃO & t/dia & kg/hab./dia \\
\hline NORDESTE & 55.794 .707 & $22.162 .000,00$ & 0,397206 \\
PERNAMBUCO & 8.796 .032 & $3.493 .837,89$ & 0,39721 \\
GARANHUNS & 135.138 & $37.505,09$ & 0,278 \\
\hline
\end{tabular}

Fonte: ABRELPE, 2013. Adaptação SILVA, R.C. (2015).

Os quantitativos revelam o quanto se faz necessário uma Gestão Integrada de RCC e uma Política que vise às diretrizes de geração, coleta, transporte e destino final adequado segundo a resolução CONAMA 307/2002.

Pinto (1999) destaca dois tipos de Gestão de RCC para municípios de médio e grande porte, a Gestão Corretiva e a Gestão Diferenciada. Nesta análise ele conclui que a forma mais adequada, ou a melhor Gestão para essas cidades é a chamada Gestão Diferenciada, esta destaca que tais cidades poderão consubstanciar a junção de menores custos de limpeza urbana, diminuição de impactos ambientais e ganhos na questão de saúde pública e ainda adquirir subsídios de substituição de agregados convencionais por agregados reciclados.

O gráfico da figura 3 evidencia uma receita destinada para a terceirização do serviço de coleta no município, visto que em 2013 a prefeitura local pagou $R \$ 59,99$ por $t$ coletada, e em 2014 houve um reajuste de $8 \%$ no valor, isso fica claro o quanto é importante para o município estabelecer diretrizes paro o gerencialmente desse material. 
Figura 3: Custo anual para coleta de RCC

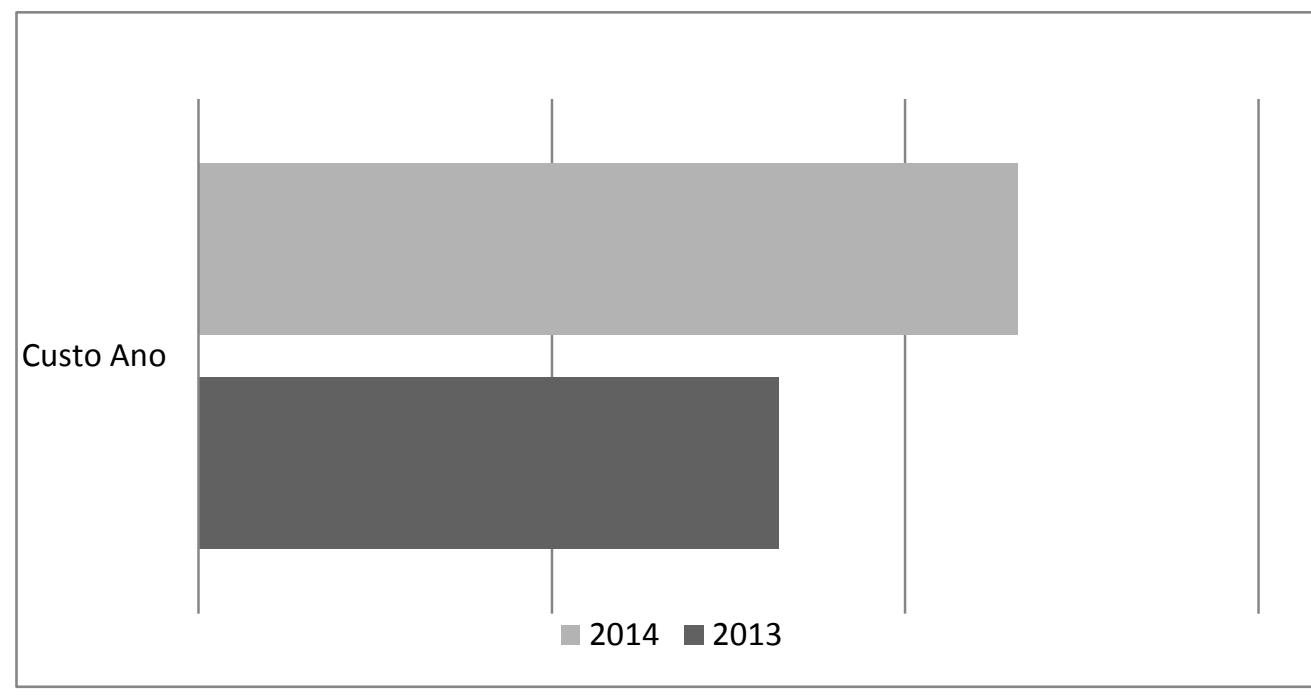

Fonte: Secretaria de Obras de Garanhuns-PE, (2014). Adaptação SILVA, R.C. (2015).

Esses dados demonstram o quanto desse material deveria ser colhido, tratado e reaproveitado. Levando em conta o altíssimo valor pago por sua coleta, o mesmo poderia ser reutilizado em pequenas obras do município viabilizando uma Gestão Municipal Diferenciada.

A implantação de uma central de tratamento e reciclagem de resíduos iria possibilitar a reciclagem de fragmentos de alvenaria de componentes cerâmicos; fragmentos de alvenaria de blocos de concreto; fragmentos de concreto, armado ou não, sem fôrmas; fragmentos de lajes e de pisos; argamassas de cal, de cimento ou mistas, de assentamento ou revestimento; componentes de concreto ou cerâmicos: blocos, tijolos, telhas, tubos, briquetes, lajotas para laje etc., também fragmentos de pedra britada e de areia naturais, sem presença significativa de terra ou outros materiais proibidos (classificação Classe A - CONAMA nํ. 307).

\section{Coleta e transporte}

A coleta e transporte de RCC no município de Garanhuns-PE é feita principalmente por meio de caminhões poliguindaste e caçambas. De acordo com 
Revista Nacional de Gerenciamento de Cidades

Figura 5: Área de descarte inadequado nas Margens da BR 424 - Magano/ Garanhuns-PE.

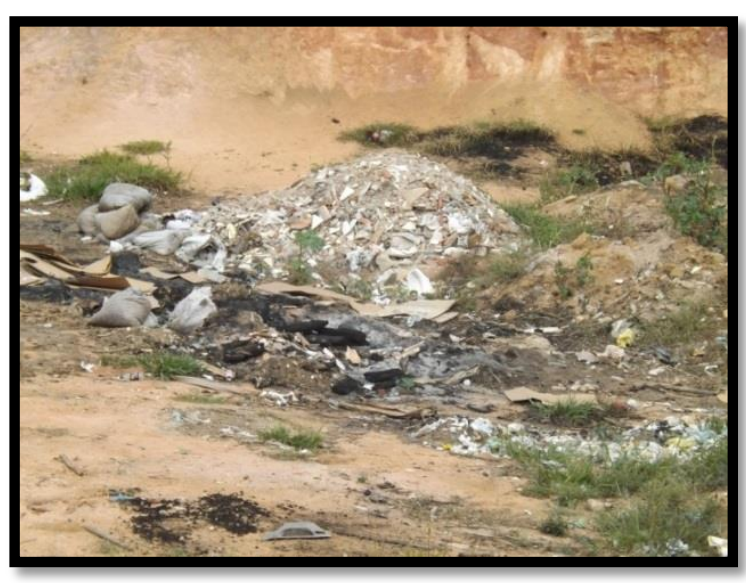

Fonte: SILVA, R. C. (2015).

Torna-se cada vez mais frequente encontrar áreas inadequada onde são depositados os entulhos, as mesmas causa a degradação do meio ambiente e da qualidade de vida da população. Ainda podemos citar poluição visual, proliferação de vetores de doenças, entupimento de galerias e da rede de drenagem entre outros. A deposição irregular está vinculada "tanto à ação de pequenos geradores e coletores, quanto à atuação de empresas coletoras para médios e grandes geradores" (PINTO, 2008).

\section{CONCLUSÃO}

A Gestão de Resíduos da Construção Civil pode e deve ser uma prática integrada e sustentável entre o setor público e privado.

$O$ desafio deve ser enfrentado por todos os agentes que fazem parte desse processo: pequenas e grandes construtoras, ou seja, os geradores desse material, coletores, transportadores e o Poder Público. 
O maior problema está ligado ao quantitativo produzido e a disposição no meio ambiente sem nenhum cuidado ou tratamento, causando impactos na saúde pública e ambiental.

A partir da Resolução CONAMA, o poder público e o privado deve integrar uma gestão sustentável dos RCC, responsabilizando de fato os agentes envolvidos. É seguindo essas normatizes que se faz urgente à criação de uma política integrada a esses setores, visando uma melhor coleta, transporte e destino correto desses materiais.

\section{REFERENCIAS BIBLIOGRÁFICAS}

ABRELPE - Associação Brasileira de Empresas de Limpeza Pública e Resíduos Especiais. Panorama dos Resíduos Sólidos no Brasil 2013.

BRASIL. Associação Brasileira de Normas Técnicas-ABNT/Norma Brasileira Regulamentadora-NBR - 10004(2004). Dispõe da Classificação dos Resíduos Sólidos. ABNT NBR 10004:2004.

BRASIL. DA POLÍTICA NACIONAL DO MEIO AMBIENTE. LEI 6.938/1981 (LEI ORDINÁRIA) 31/08/1981. Dispõe sobre a Política Nacional do Meio Ambiente, seus fins e mecanismos de formulação e aplicação, e dá outras providências. Disponível em: http://www.planalto.gov.br/ccivil_03/leis//6938.htm. Acesso em 02/2015.

BRASIL. Decreto Federal no 99.274/1990. Regulamenta a Lei no 6.902, de 27 de abril de 1981, e a Lei $n$ o 6.938, de 31 de agosto de 1981, que dispõem, respectivamente sobre a criação de Estações Ecológicas e Áreas de Proteção Ambiental e sobre a Política Nacional do Meio Ambiente, e dá outras providências. Disponível em: http://www.planalto.gov.br/ccivil_03/decreto/antigos/d99274.htm. Acesso em 02/2015.

BRASIL. IBGE - Instituto Brasileiro de Geografia e Estatística. Disponível em:http://www.ibge.gov.br/home/. Acesso em 08/2014.

BRASIL. Ministério do Meio Ambiente, Conselho Nacional do Meio Ambiente. Resolução 307, de 05 de julho de 2002. Art. 2을 Inciso I, Art. 3‥ Estabelece diretrizes, critérios e procedimentos para a gestão dos resíduos da construção civil. Diário Oficial da República Federativa do Brasil, Brasília, DF, no 136, 17 de julho de 2002. Seção 1, p. 95-96.

BRASIL. Ministério do Meio Ambiente, Conselho Nacional do Meio Ambiente. Resolução № 448, de 18 de janeiro de 2012. . Estabelece diretrizes, critérios e procedimentos para a gestão dos resíduos da construção civil. Diário Oficial da República Federativa do Brasil, Brasília, DF, nº 14, 19 de janeiro de 2012.

BRASIL. RESOLUÇÃO № 307, DE5 DE JULHO DE 2002. Publicada no DOU oํ 136, de 17/07/2002, pp. 95 e 96 - Alterada pelas Resoluções 348, de 2004, no 431, de 2011, e no 448/2012. 
